\title{
Dengue in Children: Prolife of Hematological and Biochemical Parameters
}

\author{
Chhaya Gupta*, Neeru Gupta, Jatin Munjal and Shephali Sharma \\ Sri Balaji Action Medical Institute, Paschim vihar, New Delhi, India
}

\begin{abstract}
Background: Dengue is the most important emerging tropical viral disease in the world today. The World Health Organisation[WHO] estimates 50 million dengue infections occur annually and almost half the world's population lives in countries where dengue infection is endemic1. Over the past several years, the epidemiological profile of dengue has been changing progressively and is currently characterized by an increase in the number of cases in children under 15 years of age 2 .
\end{abstract}

Aim and objective: To evaluate and co-relate the hematological and biochemical data in children during the evolution of dengue fever and to predict the severity of the disease.

Methods: It is a retrorespective study of 30 seropositive dengue cases below 18 years of age carried out in the period of Jan 2016 to Nov 2016. Their hematological and biochemical parameters were studied at Sri Balaji Action Medical Institute, New Delhi.

Conclusion: Awareness, early recognition and early diagnosis are important for favorable out come. Hematological and biochemical profile are very helpful tools for disease monitoring and can be helpful in prediction of prognosis. Thrombocytopenia is a feature of dengue.

Keywords: Dengue, Children, Complete Hemogram, Biochemical Profile.

\section{Introduction}

Dengue is an acute febrile disease. The etiologic agent of dengue is the dengue virus [DENV], which belongs to the Flaviviridae family and the Flavivirus genus and has four serotypes [DEN-1, DEN - 2, DEN - 3, DEN - 4]. Dengue is the most common arthropod borne viral disease transmitted by mosquito of the genus Aedes aegypti and is one of the most significant diseases because it is associated with high rates of morbidity and mortality ${ }^{[1,2,3 .]}$

This disease currently represents a major public health issue especially in tropical and subtropical countries, where environmental conditions enable the development and proliferation of mosquito which breeds exclusively in domestic man made water receptacles ${ }^{[2,4]}$.

Dengue shows a cyclical trend with a peak in September, October, November every year.It is a disease with a wide clinical spectrum and a wide variety of presentation, ranging from asymptomatic to an undifferentiated fever (viral syndrome) to the more severe forms such as severe dengue (SD) or Dengue Haemorrhagic Fever (DHF) ${ }^{[5]}$. The WHO organization revised it's case definitions in 2009 and now distinguishes as Dengue fever, Dengue fever with warning signs and severe dengue fever ${ }^{[1]}$.In children dengue can be asymptomatic or polysymptomatic,which may hinder the differential diagnosis ${ }^{[2]}$
The study by RochaLA etal observed the changing epidemiological trend in Brazil with shift being observed in the age group of the affected population ${ }^{[6]}$.There are few reports on the clinical and laboratory differences in children and adults with dengue ${ }^{[2,7,8]}$

The intial febrile illness begins with rapid onset of high grade fever which is accompanied by retroorbital headache, severe myalgia, arthralgia, nausea, vomiting (more common in children) and general fatigue. A confluent maculopapular rash (more common in children) appear during end of febrile stage. Petechiae and purpura indicate capillary fragility ${ }^{[8]}$

Although most children recover directly after the intial stage, a small proportion will develop systemic capillary leakage. Delayed diagnosis of severe dengue is associated with high mortality(up to $40 \%$ ). Therefore warning signs for deterioration are of utmost clinical importance. ${ }^{[2][8]}$

\section{Material and Methods}

This is a descriptive, observational retrospective study of secondary data obtained from the medical records of 30 patients under the age of 18 who had serological diagnosis of dengue fever at Sri Balaji Action Medical Institute, New Delhi, India.conducted in period January to November 2016. The cases included in the study showed positive dengue NS1 (Non structural proteins-1 antigen) or IgM 
serology performed byEnzyme Linked Immunosorbant Assay (ELISA).

Complete haemogram including haemoglobin, haematocrit(Hct), total leucocyte count, differential count and platelet count were noted. Haemogram profile was done on Automated Hematology analyser sysmex XN1000. Peripheral smear examination was done in all the patients.

Biochemical parameters like Aspartate Transaminase (AST), Alanine Transminase ( ALT), total protein, Albumin and globulin were done by spectrophotometric method done on Cobas c501.

Hemoglobin of patients were grouped as $<10 \mathrm{gm} / \mathrm{dl}, 10-$ $15 \mathrm{gm} / \mathrm{dl},>15 \mathrm{gm} / \mathrm{dl}$. Haematocrit was grouped as $<35 \%$, $35-45 \%,>45 \%$. The hematocrit parameter to evaluate hemoconcentration was $\mathrm{Ht}>38 \% \%^{[2]}$. Total leucocyte counts of the patients were gouped as $<4000,4000$ to 11000 , $>11000$ cells/cumm. Leucopenia was defined as total leukocyte count $<4000$ cells/cumm. Lymphocytosis was defined as $>45 \%$ lymphocytes in the differential count.

Thrombocytopenia was defined as a platelet count of $<150000 / \mu 1$. Platelet counts of the patients were grouped as $<20,000 / \mu 1,21,000-50,000 / \mu 1,50000-1 \mathrm{lakh} / \mu 1,1-1.5$ lakh $/ \mu 1,>1.5 \mathrm{lakh} / \mu \mathrm{l}$.

AST result $>50 \mathrm{IU} / \mathrm{L}$ and $\mathrm{ALT}$ result $>50 \mathrm{IU} / \mathrm{L}$ were considered to be high.

Hypoalbuminemia was defined as an albumin level of less than $3.4 \mathrm{~g} / \mathrm{dL}$ for patients 7 months or older and less than $2.5 \mathrm{~g} / \mathrm{dL}$ for patients younger than 7 months. ${ }^{[9]}$ Patients diagnosed to have co-infection with malaria, chickungunya or enteric fever by relevant investigation were excluded from the study.

\section{Result}

In a study period of Jan to Nov 2016, We have 214 seropositive Dengue cases, out of which 35 (16.3\%) were children. One case showed co-infection with chikungunya and two showed co- infection with enteric fever.Two cases were on OPD basis whose details were not available. Therefore these 5 cases were excluded from the study.

Month wise distribution of cases is as depicted in Fig1. There is a surge in the number of cases in the monsoon period i.e September, October and November. The presenting symptoms of the patients are shown in Fig2. Fever, nausea -vomiting and abdominal pain were the most frequent complaints. The pattern of dengue seropositivity is depicted in Fig3.

Anemia with haemoglobin $<10 \mathrm{gm} / \mathrm{dl}$ was observed in $10 \%(3 / 30)$ cases. Seventy percent of cases had haemoglobin in range of $10-15 \mathrm{gm} / \mathrm{dl}$. Laboratory investigation revealed that the most common haematological abnormality was platelet count $<1,00,000 / \mu 1$ and hemoconcentration [Hct $>38 \%$ ] which was observed in $22(73.3 \%)$ patients. Thirty six percent of patients had platelet count between $20,000-50,000 / \mu 1$. and thirty three percent cases had platelet count of $<20,000 / \mu 1$. [Table-1]Hct of the patients are grouped in Table2. Leucopenia was seen in $8(26.6 \%)$ patients however normal counts were observed in 16 $(53.3 \%)$ of cases[Table-3]. Leucopenia with lymphocytosis were seen in $4(13 \%)$ of cases.

Liver function tests (LFT) showed increased levels of aspartate aminotransferase (AST) and alanine aminotransferases (ALT). AST was raised in $24(85 \%)$

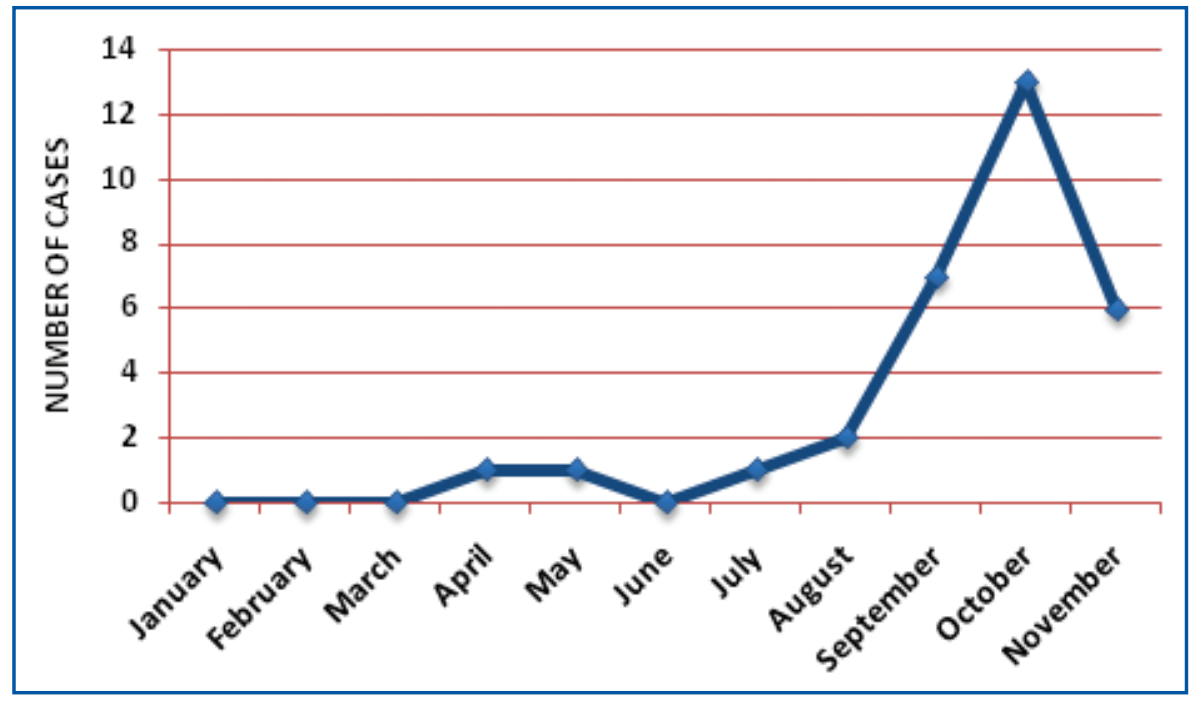

Fig. 1: Month wise distribution of dengue cases. 


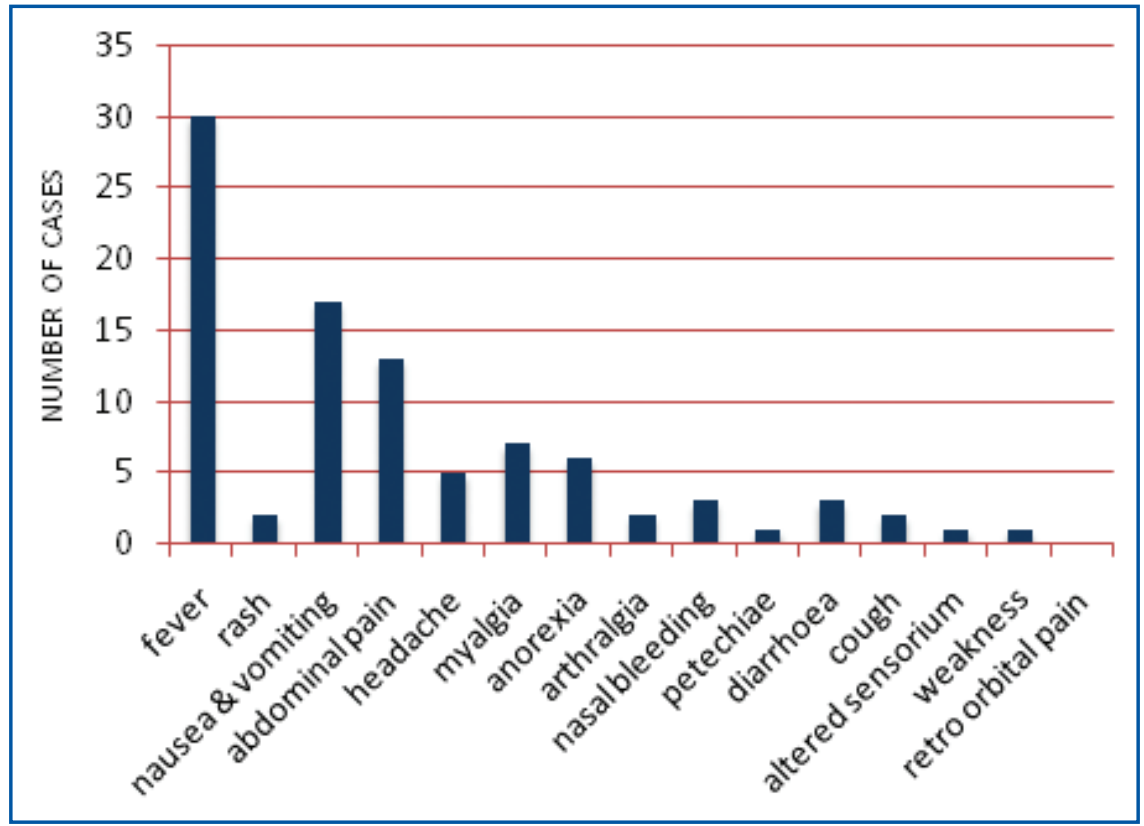

Fig. 2: Presenting clinical symptoms of the patients.

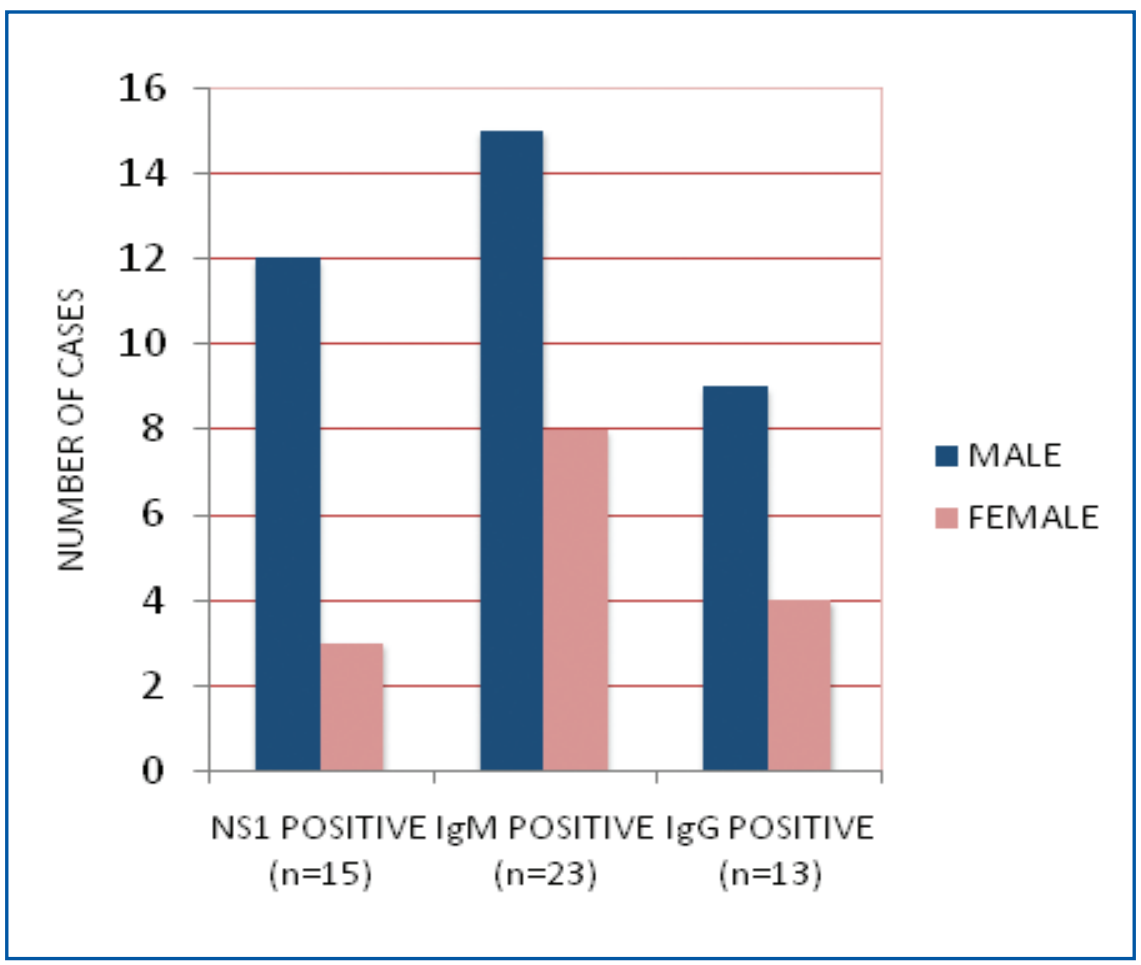

Fig. 3: Pattern of dengue seropositivity.

patients . Mean and median AST levels were 1393.3 IU/1 and 165 IU/1 respectively. Maximum dearangement observed was 24,874 IU/1 which was 620 times the cut off valve. ALT was raised in $23(82.1 \%)$ patients $(n=30)$. Alkaline phosphatase levels ranged from 56 to $421 \mathrm{IU} / 1$ $(\mathrm{n}=14)$. Total protein was $<6 \mathrm{~g}$ in $8(42 \%)$ patients $(\mathrm{n}=19)$ and albumin $<3.5 \mathrm{~g}$ in $7(36.8 \%)$ patients $(\mathrm{n}=19)$.

There was one mortality due to the disease. The patient had severe thrombocytopenia, markedly raised AST and ALT and deranged Prothrombin Time. 
Table 1: Platelet profile of patients.

\begin{tabular}{|c|c|c|}
\hline Platelet count $($ per $\mu \mathrm{l})$ & Results $(\mathrm{n}=30)$ & 33 \\
\hline$<20,000$ & 10 & 36.6 \\
\hline $21000-50000$ & 11 & 3.3 \\
\hline $50000-1$ lakh & 1 & 13.3 \\
\hline $1-1.5$ lakh & 4 & 13.3 \\
\hline
\end{tabular}

Table-2: Haematocrit $\%$ of patients.

\begin{tabular}{|c|c|c|}
\hline Haematocrit (\%) & Results $(\mathrm{n}=30)$ & 13.3 \\
\hline$<35$ & 4 & 60 \\
\hline $35-45$ & 18 & 26.6 \\
\hline
\end{tabular}

Table 3: Total leucocyte profile of patients.

\begin{tabular}{|c|c|c|}
\hline Total Leucocyte Count $\left(\right.$ cells $\left./ \mathrm{mm}^{3}\right)$ & Results $(\mathrm{n}=30)$ & 26.6 \\
\hline$<4000$ & 8 & 53.3 \\
\hline $4000-11000$ & 16 & 20 \\
\hline
\end{tabular}

\section{Discussion}

The first clinical case report of dengue was from Philadelphia by Berjanin Rush, who coined the term "Break Bone Fever" because of the symptoms of myalgia and arthralgia [10]. In india ,dengue was first reported in 1946, DENV-1 was first reported in 1956 at vellore,DENV - 2 in

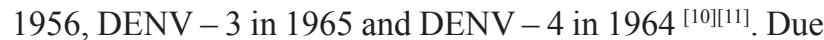
to changing climate, urbanization poor living conditions and inadequate waste management, vector borne disease like dengue fever are becoming more common. Although vector control programs are launched in endemic countries every year, yet dengue fever has become a serious problem worldwide. India being a tropical country provides suitable weather for Aedes mosquito to grow and an increase in the disease burden has been noticed in recent years ${ }^{[12]}$.

In our study there was a slight male preponderance. Similar observations have been made by De souza LJ et $\mathrm{al}^{[2]}$, Patel $\mathrm{K}$ et al ${ }^{[13]}$ and Dongre $\mathrm{T}$ et al ${ }^{[14]}$. Hypothetically this can by related to the increased risk of exposure of male gender to mosquito bites due to social, occupational and recreational activities ${ }^{[13]}$.

The most significant laboratory abnormality seen in our patients was thrombocytopenia as also observered by Azin et $\mathrm{al}^{[5]}$, De souza et $\mathrm{al}^{[2]}$ Dongre et al ${ }^{[14]}$ and patel et $\mathrm{al}^{[13]}$.Haemorrhagic manifestation are very common with severe thrombocytopenia and severity of haemorrhagic tendency correlates with platelet counts. At times automatic counter gives a false low reading in case of large platelets. Such cases can be obviated by assessment of platelets on smears. Leucopenia was observed in $26.6 \%$ of patients however Patel et al ${ }^{[13]}$ observed $56.92 \%$ patients with leucopenia. Leucopenia is due to the direct marrow suppression by the virus.

Patel et $\mathrm{al}^{[13]}$ observed increase in concentration of atypical lymphocytes. It mainly represents that serum immunoglobulin production is enhanced during dengue viral infection, these are mostly against the specific serotype and obviously not protection to the infections caused by other serotypes ${ }^{[15]}$.

LFT showed increased levels of AST and ALT in > $80 \%$ of our cases which was comparable to $100 \%$ in a study by Bhaskar et al. ${ }^{[16]}$ and $90 \%$ by Kuo et al ${ }^{[17]}$. In $42.8 \%$ of children AST levels were more than five times indicating that they are at higher risk of hepatic involvement and developing hepatic encephalopathy ${ }^{[16]}$. Fourty two percent of our patients showed total protein of $<6$ gm and $36.8 \%$ of patients had $<3.5 \mathrm{gm}$ albumin however it was different from Bhasker et al. They observed Hypoproteinemia with hypoalbuminemia in $74 \%$ of cases. Hypoalbaminemia is an important factor of fluid loss into third space which is indicative of severity of Dengue infection ${ }^{[16]}$ Dengue fever is a self limiting disease. Dengue fever evolves with laboratory alteration starting on the $3^{\text {rd }}$ day and becoming most evident on the $5^{\text {th }}$ day with values restored to normal by $11^{\text {th }}$ day.$^{[5]}$ Dengue haemorrhagic fever causes morbidity and mortality. No antiviral treatment is available hence fluid and electrolyte replacement and supportive therapy are the available modalities of treatment. Due to lack of specific treatment it is important to implement public health policies and vector control measures to curtail the diseases ${ }^{[18]}$

\section{Conclusion}

Due to lack of an effective dengue vaccine as well as to the absence of targeted treatment options, the knowledge 
and skills to recognize and diagnose the disease before it reaches its' critical phase are of utmost importance. The complete haemogram is the most important guide to therapy and prognosis along with liver function test.

\section{References}

1. World Health organization 2009 Dengue: Guidelines for Diagnosis, treatment, Prevention and control, New edition, World Health Organization and TDR For research in diseases of poverty.

2. Luiz Jose de Souza, Lais Bastos Pessanha, Laura C. Mansur, LA de souza, MBT Ribeiro, Monique do Vda Silveira, Jaoo TDS filho. Comparisen of clinical and laboratory characteristics between children and adults with dengue. Braz J of infections diseases. 2013: 17 (1): $27-31$.

3. Chambers, TJ, Hahn, CS, Galler, R, \& Rice, CM. "Flavivirus genome organization, expression, and replication.” Annu Rev Microbiol.; 44: 649-88, 1990.

4. Jain A, Shah AA,Patel P,Desai M, Somani S, Parikh P et al. A clinico - hematological profile of dengue outbreak among healthcare professionals with analysis on economic impact. National Journal of Community medicine; vol 4, issue 2, April - June 2013.

5. Francisaca Raimunda F, Guerreiro A Zin, Romelia Pinheiro Goncalves, Maria $\mathrm{H}$ dasilva Pitombeira, DM lima, I C Branco. Dengue: profile of hematological and biochemical dynamics. Rev Bras Hematol Hemoter, 2012; 34 (1): 36 41.

6. Rocha LA, Tavil PL, Dengue in children : clinical and epidemiological characteristics, Manus, state of Amazonas, 2006 and 2007. Rev Soc Bras Med Trop. 2009; 42: 18 - 22 [in Portuguese]

7. Wang C, Lee I, Sum, etal. Deffernces in clinical and laboratory characterstion and disease severity between children and adults with dengue virus infection in Taiwan, 2002. Trans RSoc Tro med Hyg, 2009: 103 : 871-7.
8. Roland elling, philipp Henneke, christophhatz, Markus Hufnagel. Dengue fever in children where are we now? The pediatric infections disease journal. Vol 32, No 9, September 2013, 1020-22.

9. Ira N. Horowitz, Kenneth Tai,Hypoalbuminemia in Critically Ill Children.Arch Pediatr Adolesc Med. 2007;161(11):10481052. doi:10.1001/archpedi.161.11.1048

10. Gupta N,Srivastava S,Jain A,Chaturvedi UC.Dengue in India.Indian J Med Res.2012;136:373-90

11. Neeraja M,Lakshmi V,Dash PK,Parida MM,Rao PVL.The clinical,serological and molecular diagnosis of emerging dengu infection at a tertiary care institute in southernIndia. Journal of clinical and diagnostic Research.2013;7:457-461.

12. Munde DD,Shetkar UB.Clinical features and hematological profile of dengue fever,Indian Journal of Applied research;Jan2013; vol3;issue1:131-132.

13. Patel K, Patel D, Das V.K. Hematological Parameters and Its Utility in Dengue fever : A prospective study. International Journal of Science and Research (IJSR), Vol 5 issue 4, April 2016.

14. Dongre T, Karmarkar P. Hematological Parameters and its utility in dengue - A prospective study. IOSR Journal of dental and medical sciences, Vol 14, issue 2 ver. II (feb. 2015), PP 31 - 34.

15. Wilder-Smith A,Schwartz E.Dengue in travelers .N Engl J M.2005;353:924-32.

16. Bhaskar I, Bhushan A, Maitty C. Evaluation of the hepatic function in dengue patients in Kolkata. J Invest Biochem. 2014. Vol 3. Issue 3, $129-132$.

17. Kuo CH,Tai DI,Chang-Chien CS,Liaw YF.Liver biochemical tests and dengue fever.Am J Trop Hyg.1992;47:265-70.

18. Singla N, Chaudhary P, Thakur M, Chander J. Dengue: An analysis of epidemiological pattern over a six year period. Journal of clinical and diagnostic research, 2016 Dec. Vol 10(12): DC 12 - DC 14.

*Corresponding author:

Dr. Chhaya Gupta, C-6/115A, Lawrence Road, Keshavpuram, NEW DELHI-110035 INDIA

Phone: +91 9910992059

Email: chhaya.doc_delhi@rediffmail.com

Financial or other Competing Interests: None. 